ISSN 0103-9954

\title{
EFEITO DE LODO DE PAPEL RECICLADO SOBRE O CRESCIMENTO EM DIÂMETRO DE COLO DE Eucalyptus saligna Smith.
}

\author{
EFFECT OF SLUDGE RECYCLED PAPER ON STEM DIAMETER GROWTH OF \\ Eucalyptus saligna Smith.
}

\author{
Alvaro Boson de Castro Faria ${ }^{1}$ Celso Garcia Auer ${ }^{2}$ Alessandro Camargo Ângelo ${ }^{3}$ \\ Epitágoras Rodson Oliveira Costa ${ }^{4}$
}

\begin{abstract}
RESUMO
Os resíduos sólidos da indústria de reciclagem de papel têm potencial de uso para correção da acidez do solo e como fonte de nutrientes para as culturas agrícolas e florestais. Este trabalho teve como objetivo avaliar os efeitos do lodo de papel reciclado sobre o crescimento de diâmetro de colo de Eucalyptus saligna Smith, com base em um delineamento fatorial $4 \times 2 \times 5$, sendo os fatores a dose do lodo, a adubação de cobertura e os dias após o tratamento, com 16 mudas implantadas em vasos a campo, durante 120 dias. Apesar de seu promissor uso como corretivo de solo, o lodo de papel reciclado prejudicou o desenvolvimento das plantas, e a maior dose prejudicou a adubação em cobertura.
\end{abstract}

Palavras-chave: produção florestal; resíduos agroindustriais; sustentabilidade.

\begin{abstract}
The solid waste of recycling paper industry has potential to be used for the correction of soil acidity and as a source of nutrients for agricultural crops and forestry. This study aimed to evaluate the effects of recycled paper sludge on the stem diameter growth of Eucalyptus saligna Smith, based on a 4x2x5 factorial design, being the factors the dose of the sludge, the top fertilization and the days after treatment, during 120 days. Despite its promising use as corrective of soil, sludge recycled paper hindered the development of stem diameter, and the highest dose damaged the fertilization.
\end{abstract}

Keywords: forestry; agro-industrial residues; sustainability.

\section{INTRODUÇÃO}

O setor de papel e celulose ocupa lugar de destaque no Brasil, colocando o país entre os principais produtores mundiais. A produção de resíduos pelas empresas, é inerente aos processos industriais de transformação de matérias primas. As fábricas dos chamados papéis tissue, que são produzidos a partir de matéria prima reciclada, são grandes produtoras de resíduos.

Por outro lado, os passivos ambientais destas indústrias podem ser reutilizados como insumos para a geração de produtos inovadores, contribuindo para o desenvolvimento de tecnologias mais limpas, para a geração de empregos e renda em novos mercados. Atualmente, um dos grandes desafios das indústrias de papel é a disposição final dos resíduos, hoje destinados a aterros industriais.

1 Engenheiro Florestal, Dr., Professor Adjunto de Engenharia Florestal, Universidade Tecnológica Federal do Paraná, Estrada para Boa Esperança, km 4, CEP 85660-000, Dois Vizinhos (PR), Brasil. alvarob@utfpr.edu.br

2 Engenheiro Florestal, Dr., Pesquisador da Embrapa Florestas, Km 111 da Estrada da Ribeira, Caixa Postal 319, CEP 83411-000, Colombo (PR), Brasil. celso.auer@embrapa.br

3 Engenheiro Florestal, Dr., Professor Associado do Centro de Ciências Florestais e da Madeira, Universidade Federal do Paraná, Rua Lothário Meissner, 900, Jardim Botânico, CEP 80210-170, Curitiba (PR), Brasil. alessandrocangelo@gmail.com

4 Engenheiro Agrônomo, MSc., CVG Cia Volta Grande Papel, Distrito de Volta Grande, CEP 89295-000, Rio Negrinho (SC), Brasil. epitagorasc@gmail.com

Recebido para publicação em 18/06/2012 e aceito em 13/02/2015 
Desde a década de 1990, pesquisas são feitas com cinzas de caldeira e resíduo celulósico (FERREIRA et al., 1995; BELLOTE et al., 1998; ANDRADE et al., 2003). Segundo Balbinot Júnior et al. (2006b), o lodo de papel e do papel reciclado são ricos em óxidos de cálcio e magnésio e tem potencial para uso como corretivo de solos, tornando-os interessantes para serem aproveitados em culturas implantadas em solos com elevada acidez.

Há escassez de trabalhos sobre o uso de resíduos de reciclagem de papel para correção da acidez do solo e/ou como fonte de nutrientes para as culturas agrícolas eflorestais (BALBINOT JÚNIOR. et al., 2006a). Corretivos de solo podem causar a redução dos teores de $\mathrm{K}^{+}$no solo e a calagem para plantações de eucalipto pode ser desaconselhável, até que se conheça a sua implicação com os demais nutrientes e com as necessidades da planta (VALERI et al., 1985).

Dentro deste contexto objetivou-se avaliar o efeito de lodo de papel reciclado no solo, no crescimento e na nutrição de Eucalyptus saligna Smith.

\section{MATERIAL E MÉTODOS}

\section{Local de estudo e avaliações preliminares}

O estudo foi realizado em área pertencente à Universidade Estadual do Centro Oeste (Unicentro), no município de Irati (PR), na região do Segundo Planalto Paranaense, com clima tipo $\mathrm{Cfb}$ segundo a classificação de Köppen, com geadas frequentes no inverno.

Foi utilizado o latossolo com textura

TABELA 1: Caracterização química do resíduo de lodo de papel reciclado.

TABLE 1: Chemical characterization of the recycled paper sludge residue.

\begin{tabular}{lc}
\hline \multicolumn{1}{c}{ Característica } & Resíduo \\
\hline Umidade $(\mathrm{g} / 100 \mathrm{~g})$ & 65 a 66 \\
$\mathrm{pH}$ & 7,3 a 7,6 \\
Carbono orgânico $(\mathrm{g} / 100 \mathrm{~g})$ & 13 a 15 \\
Nitrogênio $(\mathrm{TKN})(\mathrm{g} / 100 \mathrm{~g})$ & 0,19 a 0,28 \\
$\mathrm{P}_{2} \mathrm{O}_{5}$ total $(\mathrm{g} / 100 \mathrm{~g})$ & 0,16 a 0,18 \\
$\mathrm{~K}_{2} \mathrm{O}$ total $(\mathrm{g} / 100 \mathrm{~g})$ & 0,11 \\
CaO total $(\mathrm{g} / 100 \mathrm{~g})$ & 13 a 22 \\
$\mathrm{MgO}$ total $(\mathrm{g} / 100 \mathrm{~g})$ & 0,42 a 0,83 \\
Enxofre total $(\mathrm{g} / 100 \mathrm{~g})$ & 0,07 a 0,17 \\
Cobre total $(\mathrm{mg} / \mathrm{kg})$ & 91 a 176 \\
Zinco total $(\mathrm{mg} / \mathrm{kg})$ & 132 a 321 \\
Ferro total $(\mathrm{g} / 100 \mathrm{~g})$ & 0,22 a 0,28 \\
Manganês total $(\mathrm{mg} / \mathrm{kg})$ & 55 a 59 \\
Sódio total $(\mathrm{g} / 100 \mathrm{~g})$ & 0,45 a 0,66 \\
Boro total $(\mathrm{mg} / \mathrm{kg})$ & 11 a 17 \\
Chumbo total $(\mathrm{mg} / \mathrm{kg})$ & 23 a 27 \\
Níquel total $(\mathrm{mg} / \mathrm{kg})$ & $<5$ a 35 \\
Cádmio total $(\mathrm{mg} / \mathrm{kg})$ & $<2$ \\
Cromo total $(\mathrm{mg} / \mathrm{kg})$ & 10 a 46 \\
Mercúrio $(\mathrm{mg} / \mathrm{kg})$ & 0,01 a 0,04 \\
Molibdênio $(\mathrm{mg} / \mathrm{kg})$ & $<15$ \\
Cobalto total $(\mathrm{mg} / \mathrm{kg})$ & 16 a 23 \\
Alumínio total $(\mathrm{g} / 100 \mathrm{~g})$ & 5,1 a 6,3 \\
Cinzas $(\mathrm{g} / 100 \mathrm{~g})$ & 71 a 72 \\
\hline Fo & \\
\hline
\end{tabular}

Fonte: Balbinot Junior et al. (2006a; 2006b) 
argilosa, para ser misturado em diferentes proporções com o resíduo de papel reciclado. O solo apresentava-se ácido, com baixos teores em potássio e sódio, teores médios de $\mathrm{Ca}, \mathrm{Mg}, \mathrm{Al}, \mathrm{MO}$ e fósforo (P) de 3,04 $\mathrm{cmol}_{\mathrm{c}} \mathrm{dm}^{-3}, 2,14 \mathrm{cmol}_{\mathrm{c}} \mathrm{dm}^{-3}, 5,36 \mathrm{cmol}_{\mathrm{c}}$ $\mathrm{dm}^{-3}, 40,54 \mathrm{~g} \mathrm{dm}^{-3}$, e $8,59 \mathrm{mg} \mathrm{dm}^{-3}$, respectivamente. A saturação por bases (V\%) média era de $22,4 \%$, contendo alumínio em nível tóxico às plantas.

Quanto ao resíduo utilizado, considerouse que este possuía as características descritas por Balbinot Junior et al. (2006a; 2006b) (Tabela 1). O resíduo de papel reciclado foi doado pela Cia. Volta Grande (CVG).

A granulometria do resíduo foi avaliada pela escala de Wentworth. O resíduo foi "esfarelado" manualmente pela fricção sobre grelhas de metal, formando agregados com diâmetro de cerca de 15 $\mathrm{mm}$ e formato pouco uniforme. No momento da implantação do experimento, o teor de umidade gravimétrica era de $60,7 \pm 3,7 \%$.

\section{Tratamentos}

As mudas utilizadas foram de Eucalyptus saligna $\mathrm{Sm}$., com três meses de idade, provenientes do viveiro da empresa Klabin. Para Garcia et al. (1991), o Eucalyptus saligna é pouco tolerante a baixa fertilidade do solo, ao déficit hídrico na estação seca e à competição entre as árvores. Já para Ferreira (1979), esta espécie é recomendada para todas as regiões, com restrições para locais com geadas ou deficiências hídricas severas.

Os tratamentos consistiram em doses crescentes de resíduo misturado com o solo com o uso de uma betoneira de construção civil. As misturas foram acondicionadas em vasos de polipropileno de 8,5 L. O plantio das mudas foi feito simulando a implantação florestal, uma vez que os vasos foram posicionados em campo em uma área plana e com espaçamento de $2 \times 2 \mathrm{~m}$. Foram testadas cinco doses de resíduos, caracterizadas como $0,0 \mathrm{v} / \mathrm{v}, 25 \mathrm{v} / \mathrm{v}$, $50 \mathrm{v} / \mathrm{v}, 75 \mathrm{v} / \mathrm{v}$ (onde $\mathrm{v} / \mathrm{v}$ significa porcentagem do volume do vaso).

O segundo fator avaliado foi o efeito da adubação em cobertura com NPK na proporção 04-14-08. Esta adubação foi realizada trinta dias após o plantio, conforme cronograma usual de implantação florestal para o eucalipto. Foi aplicado uniformemente o equivalente a $50 \mathrm{~g}$ de adubo por vaso.

Para avaliar o efeito da adubação de cobertura e da interação com as doses de resíduo, foi utilizado um delineamento fatorial $4 \times 2 \times 5$, sendo os fatores a dose do lodo, a adubação de cobertura e os dias após o tratamento (DAT), e com 16 repetições. Os dados tiveram distribuição normal e foram analisados pela Anova. As médias foram comparadas pelo teste de Tukey $(\mathrm{p}<0,05)$, usandose o software Assistat (SILVA; AZEVEDO, 2002).

Atividades silviculturais de pré e pós plantio foram realizadas, como aplicação de fosfato monoamônico dentro dos vasos (adubação de cova, no momento do plantio), controle de plantas daninhas, formigas cortadeiras, e replantio de mudas mortas (após uma semana do plantio). Foram avaliados os fatores ambientais temperatura máxima e mínima e precipitação pluviométrica durante o período das avaliações (Figura 1).

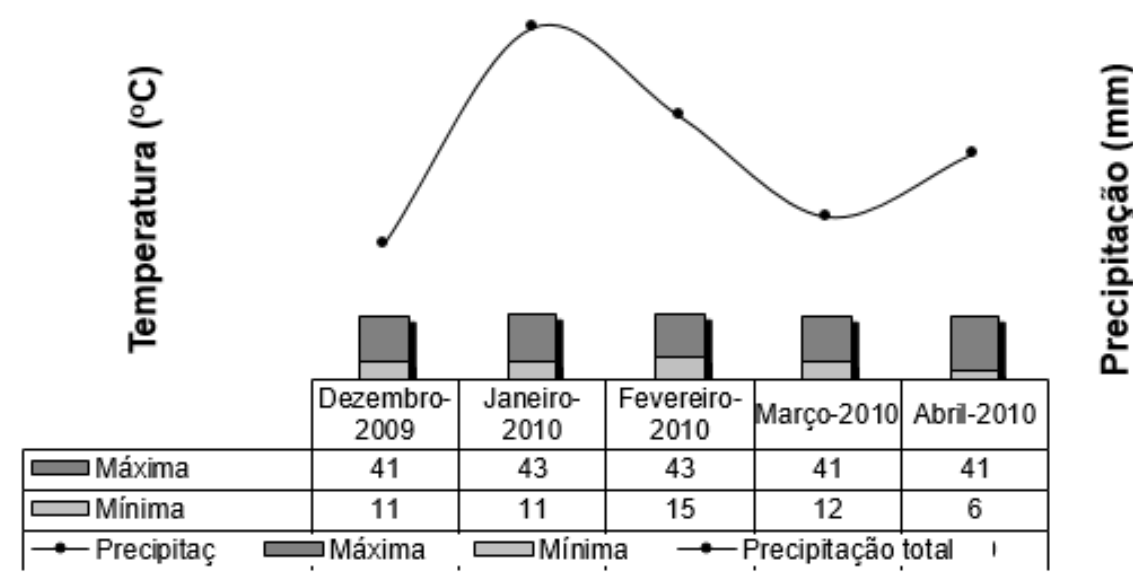

FIGURA 1: Temperatura e precipitação na área experimental.

FIGURE 1: Temperature and precipitation in the experimental site. 


\section{Crescimento}

Para a obtenção dos diâmetros de colo das plantas foram medidos com auxílio de paquímetro digital com precisão milimétrica. A medição foi tomada o mais próximo possível do solo. As alturas das mudas foram avaliadas com auxílio de régua de $50 \mathrm{~cm}$. Antes do plantio, porém, foram avaliadas vinte mudas do lote, aleatoriamente, para caracterizá-las quanto ao diâmetro de colo e altura (Tabela 2).

A implantação foi feita em dezembro de 2009 e as avaliações de crescimento foram realizadas a cada trinta dias durante 120 dias. Os dados referentes a cada parâmetro apresentaram variância homogênea. Foram analisados pela Anova e as médias comparadas pelo teste de Tukey $(\mathrm{p}<0,05)$. Elaboraram-se as tendências dos tratamentos no software Excel.

TABELA 2: Tamanho das mudas antes da implantação do experimento.

TABLE 2: Size of the seedlings before the establishment of the experiment.

\begin{tabular}{lc}
\hline & Diâmetro de colo (mm) \\
\hline Mínimo & 2,09 \\
Máximo & 3,50 \\
Amplitude & 1,41 \\
Média & 2,67 \\
Variância & 0,13 \\
Desvio Padrão & 0,36 \\
Erro Padrão da média & \\
(n=18) & 0,08 \\
Coeficiente de & \\
Variação (\%) & 0,14 \\
IC ${ }_{\text {MÁx }}$ & 2,85 \\
IC ${ }_{\text {Min }}$ & 2,49 \\
\hline Em que: IC ${ }_{\text {MÁx }}=$ Valor máximo para o Intervalo de \\
Confiança da média, ao nível de 95\% de probabilidade; \\
IC ${ }_{\text {Miñ }}=$ Valor mínimo para o Intervalo de Confiança da \\
média, ao nível de 95\% de probabilidade.
\end{tabular}

\section{RESULTADOS E DISCUSSÃO}

\section{Diâmetro de colo}

As mudas utilizadas tinham em média 2,67 mm de diâmetro de colo na implantação do experimento. Fonseca (1988) recomenda um diâmetro de colo de cerca de $2,0 \mathrm{~mm}$ para as mudas de eucalipto serem plantadas em campo, por proporcionar boa rigidez da haste. Com base neste parâmetro afirma-se que as mudas estavam adequadas para o plantio.

$\mathrm{O}$ experimento fatorial apresentou diferenças altamente significativas indicando o efeito da adubação de cobertura favorecendo o crescimento das plantas. Também houveram interações entre dose x adubação, dose x DAT, adubação $\mathrm{x}$ DAT $\mathrm{e}$ dose $\mathrm{x}$ adubação $\mathrm{x}$ DAT (Tabela 3).

Com o decorrer do tempo, notou-se a tendência na qual quanto maior a dose de resíduo presente nos vasos, menor o diâmetro das plantas. Este resultado indica que o Eucalyptus saligna é uma espécie calcífuga, que segundo SánchezBiezma, Corradine e Alvarenga (2001), é aquela que apresenta dificuldades em desenvolver-se em solos alcalinos.

Aos 60 dias após o plantio, a testemunha com adubação apresentava $6,17 \mathrm{~mm}$ de diâmetro, sendo significativamente superior a ao grupo sem adubação. Aos 90 e 120 dias após a implantação, o grupo com adubação e sem lodo também apresentaram desenvolvimento superior em relação ao grupo sem NPK. A influência positiva da adubação em cobertura no crescimento das plantas já era esperada, sendo uma prática convencional na implantação florestal do eucalipto (Tabela 4).

$\mathrm{Na}$ última avaliação (120 dias) as plantas da testemunha adubada apresentaram em média $13,76 \mathrm{~mm}$ de diâmetro e as do grupo sem adubação apresentaram $6,81 \mathrm{~mm}$, diferindo entre si estatisticamente, e sendo as doses com os melhores resultados.

Vale destacar que o período a partir de 60 dias de implantação apresentou sempre plantas significativamente superiores no grupo com adubação também para as doses $25 \mathrm{v} / \mathrm{v}$ e $50 \mathrm{v} / \mathrm{v}$ de lodo de papel reciclado. Já para a dose $75 \mathrm{v} / \mathrm{v}$, a diferença conferida pela adubação foi verificada apenas a partir de 90 dias. Assim, ficou evidenciado que o aumento da concentração de lodo dificultou o crescimento das plantas, em detrimento da adubação de cobertura.

Ressalta-se o resultado da maior dose de lodo $(75 \mathrm{v} / \mathrm{v})$ e sem adubação, que proporcionou plantas com diâmetros na ordem de 2,75 a 3,55 mm para o período de avaliações. Este grupo de plantas praticamente não se desenvolveu, uma vez que são dados com magnitudes semelhantes aos encontrados no inicio das avaliações. 
TABELA 3: Esquema fatorial do experimento.

TABLE 3: Factorial experiment.

\begin{tabular}{lcccc}
\hline \multicolumn{1}{c}{ FV } & GL & SQ & QM & F \\
\hline Dose de lodo & 3 & 676,33 & 225,44 & $271,19--$ \\
Adubação de cobertura & 1 & 129,76 & 129,76 & $156,09 * *$ \\
DAT & 4 & $1.927,55$ & 481,88 & $579,67--$ \\
Dose x Adubação & 3 & 70,62 & 23,54 & $28,31 * *$ \\
Dose x DAT & 12 & 703,11 & 58,59 & $70,48 * *$ \\
Adubação x DAT & 4 & 113,62 & 28,41 & $34,18^{* *}$ \\
Dose x Adubação x DAT & 12 & 103,36 & 8,61 & $10,36 * *$ \\
Tratamentos & 39 & $3.724,47$ & 95,49 & $114,87 * *$ \\
Resíduo & 600 & 498,78 & 0,83 & \\
\hline Total & 639 & $4.223,25$ & &
\end{tabular}

Em que: FV = Fonte de Variação; GL = Graus de Liberdade; SQ = Soma dos Quadrados; QM = Quadrado Médio; $\mathrm{F}=$ Valor $\mathrm{F}$ calculado; $--=$ Os tratamentos são quantitativos; $* *=$ significativo ao nível de $1 \%$ de probabilidade $(\mathrm{p}<0,01) ;{ }^{*}=$ significativo ao nível de $5 \%$ de probabilidade $(\mathrm{p}<0,05)$. ns não significativo $(\mathrm{p}>=0,05)$.

Diversos trabalhos sobre a aplicação de resíduos constataram o efeito significativo no crescimento de árvores e incremento de volume de madeira. Rocha et al. (2004) encontraram aumentos de produtividade de Eucalyptus grandis com o aumento das doses de lodo de esgoto aos 12, 24 e 36 meses de idade.

Com o resíduo de celulose, Rodrigues (2004) constatou que as árvores do tratamento que recebeu $80 \mathrm{t} \mathrm{ha}^{-1}$ possuíam maior biomassa de acículas, galhos, casca e lenho, favorecendo a produção em Pinus.

Barreto (2008) constatou para o diâmetro do colo de clone de Eucalyptus grandis x Eucalyptus urophylla o efeito positivo das doses de resíduo de indústria de celulose e papel, aos 120 dias após o plantio. Também, os resultados de Andrade et al. (2003) indicaram que a aplicação de $80 \mathrm{t} \mathrm{ha}^{-1}$ de resíduo celulósico em Eucalyptus dunnii de seis anos de idade, resultou num aumento de 43,5\% de volume cilíndrico de madeira, em relação à testemunha.

As pesquisas realizadas pela Embrapa Florestas mostraram aumentos significativos de produtividade em plantios de Eucalyptus grandis com a aplicação conjunta de adubos minerais e de resíduo celulósico (Bellote et al. 1998). Resultados no estímulo ao crescimento de florestamentos também são apresentados por Harrison et al. (2003), através do método de aplicação de resíduos orgânicos via aspersão.
É preciso analisar os diversos fatores, tais como a tecnologia de aplicação do resíduo, que influenciaram os diferentes resultados, uma vez que no presente experimento as plantas dos tratamentos com resíduo foram as que menos se desenvolveram. Nas pesquisas mencionadas, a aplicação foi feita superficialmente com uma quantidade préestabelecida do resíduo no solo ou via aspersão, e sendo então quantificado em $\mathrm{t} \mathrm{ha}^{-1}$. No presente estudo, houve previamente a transformação granulométrica e a regulação do teor de água do resíduo, além de ter sido incorporado no solo, antes do plantio.

Outros importantes fatores que comprometem uma comparação direta entre resultados são a dose, a idade do plantio, as diferentes espécies vegetais, o tipo do resíduo, e a presença de adubação mineral no experimento. No levantamento bibliográfico realizado, não foram encontrados resultados de crescimento em Eucalyptus em função do resíduo de papel reciclado; as informações mais próximas dizem respeito ao resíduo de indústria de celulose. Desta forma, são sempre recomendáveis novos testes que atestem e confirmem os resultados das pesquisas anteriores, especialmente aqueles que analisam a interação do resíduo versus adubação mineral.

Para se recomendar efetivamente a utilização do lodo de papel reciclado na implantação do Eucalyptus saligna, sugere-se novas pesquisas de campo, com ensaios em dosagens determinadas 
TABELA 4: Diâmetro de colo das mudas em função das doses de lodo de papel reciclado e adubação.

TABLE 4: Stem diameter of seedlings in function of recycled paper sludge doses and fertilization.

\begin{tabular}{clcc}
\hline Dose de Lodo (\%) & DAT (dias) & Sem adubação & Com adubação \\
\hline & 0 & $2,82 \mathrm{~A}$ & $2,53 \mathrm{~A}$ \\
0 & 30 & $3,51 \mathrm{~A}$ & $3,26 \mathrm{~A}$ \\
& 60 & $4,82 \mathrm{~B}$ & $6,17 \mathrm{~A}$ \\
& 90 & $5,88 \mathrm{~B}$ & $9,15 \mathrm{~A}$ \\
& 120 & $6,81 \mathrm{~B}$ & $13,76 \mathrm{~A}$ \\
\hline \multirow{2}{*}{25} & 0 & $2,77 \mathrm{~A}$ & $2,91 \mathrm{~A}$ \\
& 30 & $3,02 \mathrm{~A}$ & $3,10 \mathrm{~A}$ \\
& 60 & $3,05 \mathrm{~B}$ & $4,95 \mathrm{~A}$ \\
& 90 & $4,09 \mathrm{~B}$ & $6,51 \mathrm{~A}$ \\
& 120 & $5,38 \mathrm{~B}$ & $8,20 \mathrm{~A}$ \\
\hline \multirow{2}{*}{50} & 0 & $2,70 \mathrm{~A}$ & $2,52 \mathrm{~A}$ \\
& 30 & $2,83 \mathrm{~A}$ & $3,04 \mathrm{~A}$ \\
& 60 & $3,26 \mathrm{~B}$ & $4,25 \mathrm{~A}$ \\
& 90 & $3,28 \mathrm{~B}$ & $7,32 \mathrm{~A}$ \\
& 120 & $4,22 \mathrm{~B}$ & $9,17 \mathrm{~A}$ \\
\hline & 0 & $2,75 \mathrm{~A}$ & $2,58 \mathrm{~A}$ \\
& 30 & $3,12 \mathrm{~A}$ & $2,96 \mathrm{~A}$ \\
& 60 & $3,44 \mathrm{~A}$ & $3,52 \mathrm{~A}$ \\
& 90 & $3,29 \mathrm{~B}$ & $5,96 \mathrm{~A}$ \\
& 120 & $3,55 \mathrm{~B}$ & $8,15 \mathrm{~A}$ \\
\hline
\end{tabular}

Em que: DAT = Dias Após o Tratamento. As médias seguidas pela mesma letra não diferem estatisticamente entre si. Foi aplicado o Teste de Tukey ao nível de 5\% de probabilidade, CV\% $=19,74$.

em ton ha $\mathrm{a}^{-1}$, equivalentes a concentrações inferiores a de $25 \mathrm{v} / \mathrm{v}$ utilizada nos vasos, e com transformação prévia da granulometria e do teor de água do resíduo.

\section{CONCLUSÕES}

Apesar de seu promissor uso como corretivo de solo, o lodo de papel reciclado prejudicou o desenvolvimento das plantas, e a maior dose prejudicou a adubação em cobertura.

\section{REFERÊNCIAS BIBLIOGRÁFICAS}

ANDRADE, G. C. et al. Efeitos da adubação fosfatada e da aplicação de resíduo de celulose no crescimento de Eucalyptus dunnii. Boletim de Pesquisa Florestal, Colombo, n. 47, p. 43-54, 2003. BALBINOT JUNIOR, A. A. et al. Alteração em características químicas de um solo ácido pela aplicação de calcário e resíduos de reciclagem de papel. Revista de Ciências Agroveterinárias, Lages, v. 5, n. 1, p. 16-25, 2006 a.

BALBINOT JUNIOR, A. A. et al. Crescimento e teores de nutrientes em tecido de alface pela aplicação de calcário e resíduos de reciclagem de papel num solo ácido. Revista de Ciências Agroveterinárias, Lages, v. 5, n.1, p. 9-15, 2006b. BARRETO, V. C. M. Resíduos de indústria de celulose e papel na fertilidade do solo e no desenvolvimento de eucalipto. 2008. $64 \mathrm{f}$. Tese (Doutorado em Produção Vegetal) - Universidade Estadual Paulista, Jaboticabal, 2008.

BELlOTE, A. F. J. et al. Resíduos da indústria de celulose em plantios florestais. Boletim de Pesquisa Florestal, Colombo, n. 37, p. 99-106, 1998.

FERREIRA, C. A.; SILVA, H. D.; ANDRADE, G. C. Efecto de la aplicación de ceniza de caldera y residuo de celulosa en el suelo y en el crecimiento de Eucalyptus grandis. Bosque, Valdivia, v. 16, n. 1, p. 95-100, 1995. 
FERREIRA, M. Escolha de espécies de eucalipto. IPEF, Piracicaba, n. 47, 1979.

FONSECA, E. P. Efeito de diferentes substratos na produção de mudas de Eucalyptus grandis W. Hill ex Maiden em "Winstrip". 1988. $81 \mathrm{f}$. Dissertação (Mestrado em Engenharia Florestal) Universidade Federal de Viçosa, Viçosa, 1988.

GARCIA, C. H.; CORRADINE, L.; ALVARENGA, S. F. Comportamento florestal do Eucalyptus grandis e Eucalyptus saligna em diferentes espaçamentos. IPEF, Piracicaba, n. 179, 1991.

HARRISON, R. B. et al. Reciclagem de resíduos industriais e urbanos em áreas de reflorestamento. IPEF, Piracicaba, n. 198, p. 1-20, 2003.

ROCHA, G. N.; GONÇALVES, J. L. M.; MOURA, I. M. Mudanças da fertilidade do solo e crescimento de um povoamento de Eucalyptus grandis fertilizado com biossólido. Revista Brasileira de Ciência do Solo, Viçosa, v. 28, n. 4, p. 623-639, 2004.

RODRIGUES, C. M. Efeito da aplicação de resíduo da indústria de papel nos atributos químicos, físicos e biológicos do solo, na nutrição e biomassa de Pinus taeda L. 2004. 21 f. Dissertação (Mestrado em Agronomia) - Universidade Federal do Paraná, Curitiba, 2004.

SÁNCHEZ-BIEZMA, M. J.; ÁlVAREZ, J.; SILANES, M. E. L. Líquenes de las rocas ultramáficas en la Sierra de A Capelada. Botanica Complutensis, Madrid, v. 25, n. 1, p. 261-269, 2001.

SILVA, F. A. S. E.; AZEVEDO, C. A. V. Versão do programa computacional Assistat para o sistema operacional Windows. Revista Brasileira de Produtos Agroindustriais, Campina Grande, v. 4, n. 1, p. 71-78, 2002.

VALERI, S. V. et al. Efeitos do fósforo e calcário dolomítico no desenvolvimento inicial de Eucalyptus grandis Hill Ex Maiden plantado em um regossolo. IPEF, Piracicaba, n. 29, p. 55-60, 1985. 